\title{
Editorial
}

\section{Does the Addition of Gonadotropin-Releasing Hormone Analogs Improve the Pregnancy Rates in Intrauterine Insemination?}

Intrauterine insemination (IUI) combined with ovarian hyperstimulation $(\mathrm{OH})$ has been demonstrated to be an effective form of treatment for subfertile couples. Trials comparing different ovarian stimulation protocols combined with IUI were selected and reviewed in detail by Cantineau et al. ${ }^{1}$. A total of 43 trials involving 3,957 women were included. Seven studies $(n=556)$ were pooled comparing gonadotropins with anti-estrogens showing significant higher pregnancy rates with the former. Five studies $(n=313)$ compared anti-estrogens with aromatase inhibitors reporting no significant difference. The same could be concluded comparing different types of gonadotropins (nine studies included, $(n=576)$. Four studies $(n=391)$ reported the effect of adding a gonadotropin-releasing hormone (GnRH) agonist which did not improve pregnancy rates, although it resulted in significant higher multiple pregnancy rates. Data of three studies $(n=299)$ showed no convincing evidence of adding a GnRH antagonist to gonadotropins.

The purpose of Eskander's study was to determine if the use of a GnRH agonist can improve pregnancy and livebirth rates during superovulation and $\mathrm{IUI}^{2}$. In this prospective study, women aged 18-39 years underwent 500 cycles of superovulation/IUI with $(n=254)$ and without $(n=246)$ GnRH agonist. There were no statistically significant difference in the live-birth, ongoing, and clinical pregnancy rates per cycle for patients who received GnRH agonist and patients who did not receive GnRH agonist. Superovulation/IUI cycles using GnRH agonist produce similar pregnancy rates to superovulation/IUI cycles without using GnRH agonist.

Schmidt-Sarosi and Yerovi ${ }^{3}$ compared the use of two GnRH agonists, nafarelin acetate (NA) and leuprolide acetate (LA), in conjunction with human menopausal gonadotropins (hMGs)/human chorionic gonadotropin
(hCG) and IUI. There were no differences in: the number of days to ovarian suppression, the number of ampules of hMGs required, the number of $17-\mathrm{mm}$ preovulatory follicles, or the mean peak estradiol levels. NA is, at the least, comparable to LA in effecting pregnancy after superovulation/IUI.

A prospective randomized trial was carried out to compare the simplified ultralong protocol (ULP) and the long protocol (LP) of GnRHa for ovulation induction with IUI in patients with endometriosis 4]. In the ULP group ( $\mathrm{n}=39), 4$ weeks after a single injection of $3.75 \mathrm{mg}$ Decapeptyl had been given, daily subcutaneous (s.c.) administration of $0.1 \mathrm{mg}$ Decapeptyl was initiated and continued for $\leq 2$ weeks prior to ovarian stimulation. In the LP group $(n=41)$, daily s.c. administration of Decapeptyl $(0.1 \mathrm{mg})$ was initiated from the mid-luteal phase of the cycle preceding the stimulation cycle. After 14 days of administration, ovarian stimulation was started if pituitary desensitization had been achieved. The clinical pregnancy rate per cycle was significantly higher in the ULP group at $48.7 \%$ (19/39) compared with $26.8 \%$ $(11 / 41)$ in the LP group. In patients with stage III or IV endometriosis, the clinical pregnancy rate per cycle was significantly higher in the ULP group at $50.0 \%(10 / 20)$ compared with $19.0 \%$ (4/21) in the LP group. This study suggests that a simplified ULP of GnRHa could give better chances of achieving pregnancy in endometriosis patients undergoing IUI and that this protocol may be more useful in patients with an advanced stage of endometriosis ${ }^{4}$.

Bellver et al. ${ }^{5}$ conducted a study to assess whether GnRH agonist administration in the luteal phase improves pregnancy outcome in IUI cycles. The patients received a random administration of either a single subcutaneous injection of $0.1 \mathrm{mg}$ triptorelin (group $\mathrm{A} ; \mathrm{n}=172$ ) 8 days 
after hCG administration, or solvent only (group B; $\mathrm{n}=172$ ) at the same time. Pregnancy rate per randomized patient was similar in both groups ( 22.7 vs. $22.1 \%)$, as were clinical pregnancy, miscarriage, and ongoing pregnancy rates. The authors concluded that administration of GnRH agonist at the time of implantation does not improve the reproductive outcome of IUI cycles 5 .

Antagonists, on either a fixed or a flexible protocol, have been proven successful in suppressing luteinizing hormone (LH) rise in superovulated cycles ${ }^{6,7,8,9,10}$. Based on this fact, some investigators have administered antagonists in IUI cycles ${ }^{6,7,8,9,10}$. They prevented LH rise and showed increased pregnancy rates. For the GnRH antagonist administration group, higher pregnancy rates are observed when all RCTs that reach statistical significance are synthesized ${ }^{6}$. For both regimens (ganirelix and cetrorelix), a trend for higher pregnancy rates was observed. The results of the clinical pregnancy rates in the meta-analysis by Kosmas et al. ${ }^{6}$ are consistent with the studies done by Checa et al. ${ }^{7}$, Gomez-Palomares et al. ${ }^{8}$, Allegra et al. ${ }^{9}$, and Williams et al. ${ }^{10}$. In trying to interpret these results, the use of antagonist superovulated IUI scheme may be justified when an LH rise is expected, e.g., previous cycle LH rise, avoidance of insemination during weekend, or big follicles required ${ }^{6}$.

To assess the effects of using a reduced dose of ganirelix with oral contraceptive pretreatment, a pilot study of controlled ovarian hyperstimulation using pure folliclestimulating hormone (FSH) for IUI was conducted by Meldrum et al. ${ }^{11}$. Patients received oral contraceptive (OC; 30 microg ethinyl estradiol/150 microg desogestrel) for 14-21 days and recombinant (r) FSH (50-225 IU/day SC) was started on day 4 after OC discontinuation. Ganirelix acetate (125 microg/day) was started with a lead follicle diameter of $14 \mathrm{~mm}$. Duration of ganirelix acetate treatment was 4.0 (2-5) days. Seven subjects $(30.4 \%)$ delivered ten babies (with three twin pregnancies). Of the 16 subjects with measurement of LH on the day of hCG administration, only one was $<0.5$ $\mathrm{mIU} / \mathrm{ml}(0.4)$, and only one was $>10 \mathrm{mIU} / \mathrm{ml}$ (17.7), and that subject delivered twins. OC pretreatment afforded flexibility in scheduling while a reduced dose of ganirelix avoided excessive suppression of $\mathrm{LH}^{11}$. The excellent results in this pilot study for IUI suggest this regimen could be further evaluated for scheduling IUI and IVF cycles.

Attempting to compare the rates of premature luteinization (PL), clinical pregnancy, and cycle cancellation in ovulation induction (OI)-IUI cycles with and without the GnRH antagonist, cetrorelix, a randomized-controlled trial was undertaken by Steward et al. ${ }^{12}$ in which patients were randomly assigned to one of two OI-IUI protocols. Those in the cetrorelix arm showed a significantly reduced rate of PL and no change in clinical pregnancy or cycle cancellation rate, leading to the conclusion that GnRH antagonists can decrease the rate of PL, but appear to have no effect on pregnancy or cycle cancellation in gonadotropin OI-IUI cycles.

Stadtmauer et al. ${ }^{13}$ conducted a study to evaluate the effect of ganirelix, the GnRH antagonist, on gonadotropin OI in patients with polycystic ovary syndrome (PCOS). Patients were treated with rFSH alone (group 1) or in conjunction with ganirelix when the leading follicle was $\geq 13 \mathrm{~mm}$ (group 2) versus from the beginning of stimulation (group 3 ), followed by IUI. Data are suggestive of improved CPR in group 2 versus group 1 (33 vs. 19\%) and LBR (35 vs. $20 \%$ ), but not significantly different. Premature luteinization was highest in group 1 (21 vs. $1.8 \%$ in group 2 and $2.1 \%$ in group 3). Group 3 had the highest cancellation rate and cost without improving CPR and LBR. No differences were noted in peak serum E2, total gonadotropin dose, or days of stimulation. Adding ganirelix in a flexible protocol to gonadotropin OI cycles in women with PCOS may be beneficial by decreasing premature luteinization ${ }^{13}$.

Ertunc et al. ${ }^{14}$ conducted a trial to observe the effects of ganirelix on controlled ovarian stimulation (COS) and IUI cycles in women with PCOS. In women with PCOS and anovulatory infertility undergoing COS/IUI, rFSH therapy was started on day 3 . In women assigned to the control group $(n=47)$, treatment was continued up to the day of hCG administration. In patients assigned to receive $\mathrm{GnRH}$ antagonist $(\mathrm{n}=42)$, ganirelix was added when the leading follicle was $\geq 14 \mathrm{~mm}$. GnRH antagonist resulted in more monofollicular development, less premature luteinization, and less cycle cancellation in IUI cycles of patients with PCOS; however, the cost of stimulation increased without an improvement in pregnancy rates ${ }^{14}$.

Lee et al. ${ }^{14}$ conducted a prospective, randomized clinical trial to evaluate the effectiveness of a GnRH antagonist in preventing premature LH surge under a letrozole and gonadotropin protocol. A total of 61 patients were randomly assigned to either letrozole or gonadotropin treatment groups. These were distinguished by the absence (group I) or presence (group II) of supplementation with $0.25 \mathrm{mg}$ of cetrorelix. Compared 
with group I, the rate of premature LH surge was statistically significantly lower for group II, but the amount of gonadotropins used was statistically significantly higher.

In a meta-analysis by Kosmos et $\mathrm{al}^{6}, 521$ patients were administered a GnRH antagonist and 548 conservatively treated patients served as control subjects. Prospective trials were retrieved from Medline and Cochrane Library (last update October 2006). Six comparisons were retrieved including 1,069 patients. Higher pregnancy rates were found in the randomized controlled trials when a GnRH antagonist was added to a gonadotropin superovulated IUI protocol. A parallel trend for multiple pregnancy rates in the GnRH antagonist group was observed, although this did not reach statistical significance. The flexible regimen was widely used.

Certain issues need to be addressed by future clinical research. The role of LH rise in pregnancy outcome is controversial. Although the presence of an LH surge is associated with higher pregnancy rates in clomiphene citrate (CC) IUI cycles, the opposite is true for FSH cycles ${ }^{16}$. Interestingly, when FSH is added to a CC cycle, the favorable effect of LH surge remains ${ }^{16}$. In such a cycle, two benefits may arise. First, the combined effect of endogenous and exogenous FSH may decrease the total FSH required, and second, premature luteinization may have a positive effect on pregnancy rates.

Further research is needed to identify which group of patients will benefit from adding GnRH analogs to an IUI stimulation protocol. Older patients with short follicular phase and reduced ovarian reserve might benefit. This clinical recommendation is based on the facts that young age and number of follicles of diameter $<10 \mathrm{~mm}$ are a significant factor for high-order multiple pregnancy ${ }^{17}$, and young age and number of follicles $>15$ $\mathrm{mm}$ on the day of hCG administration are significant factors for multiple pregnancy ${ }^{18}$. Also for women with reduced ovarian reserve, premature luteinization occurs more frequently. This is due to defective production of gonadotropin surge attenuating factor $(\mathrm{GnSAF})^{19}$. On the contrary, a prolongation of follicular phase might allow for an increased number of mature follicles, which may enhance the possibility of pregnancy. In addition, patients with a previous canceled cycle because of premature luteinization are candidates for this treatment.

\section{References}

1. Cantineau AE, Cohlen BJ, Heineman MJ. Ovarian stimulation protocols (anti-oestrogens, gonadotrophins with and without GnRH agonists/antagonists) for intrauterine insemination (IUI) in women with subfertility. Cochrane Database Syst Rev. 2007; (2): CD005356.

2. Eskandar MA. Does the addition of a gonadotropinreleasing hormone agonist improve the pregnancy rate in intrauterine insemination? A prospective controlled trial. Gynecol Endocrinol. 2007; 23 (10): 551-5.

3. Schmidt-Sarosi CL, Yerovi LA Jr. The efficacy of nafarelin acetate versus leuprolide acetate in conjunction with human menopausal gonadotropins for superovulation/intrauterine insemination. Int $\mathrm{J}$ Fertil Menopausal Stud. 1994; 39 (1): 20-5.

4. Kim CH, Cho YK, Mok JE. Simplified ultralong protocol of gonadotrophin-releasing hormone agonist for ovulation induction with intrauterine insemination in patients with endometriosis. Hum Reprod. 1996; 11 (2): 398-402.

5. Bellver J, Labarta E, Bosch E, et al. GnRH agonist administration at the time of implantation does not improve pregnancy outcome in intrauterine insemination cycles: a randomized controlled trial. Fertil Steril. 2010; 94 (3): 1065-71.

6. Kosmas IP, Tatsioni A, Kolibianakis EM, et al. Effects and clinical significance of GnRH antagonist administration for IUI timing in FSH superovulated cycles: a metaanalysis. Fertil Steril. 2008; 90 (2): 367-72.

7. Checa MA, Prat M, Robles A, Carreras R. Use of gonadotropin-releasing hormone antagonists to overcome the drawbacks of intrauterine insemination on weekends. Fertil Steril. 2006; 3: 573-7.

8. Gomez-Palomares JL, Julia B, Cevedo-Martin B, Martinez-Burgos M, Hernandez ER, Ricciarelli E. Timing ovulation for intrauterine insemination with a GnRH antagonist. Hum Reprod. 2005; 2: 368-72.

9. Allegra A, Marino A, Coffaro F, et al. GnRH antagonistinduced inhibition of the premature LH surge increases pregnancy rates in IUI-stimulated cycles. A prospective randomized trial. Hum Reprod. 2007; 22: 101-8.

10. Williams RS, Hillard JB, De VG, et al. A randomized, multicenter study comparing the efficacy of recombinant FSH vs recombinant FSH with Ganirelix during superovulation/IUI therapy. Am J Obstet Gynecol. 2004; 2: $648-51$

11. Meldrum DR, Cassidenti DL, Rosen GF, Yee B, Wisot AL. Oral contraceptive pretreatment and half dose of ganirelix does not excessively suppress LH and may be an excellent choice for scheduling IUI cycles. J Assist Reprod Genet. 2008; 25 (8): 417-20.

12. Steward RG, Gill I, Williams DB, Witz CA, Griffith J, Haddad GF. Cetrorelix lowers premature luteinization rate in gonadotropin ovulation induction-intrauterine 
insemination cycles: a randomized-controlled clinical trial. Fertil Steril. 2011; 95 (1): 434-6.

13. Stadtmauer LA, Sarhan A, Duran EH, Beydoun H, Bocca S, Pultz B, Oehninger S. The impact of a gonadotropinreleasing hormone antagonist on gonadotropin ovulation induction cycles in women with polycystic ovary syndrome: a prospective randomized study. Fertil Steril. 2011; 95 (1): 216-20.

14. Ertunc D, Tok EC, Savas A, Ozturk I, Dilek S Gonadotropin-releasing hormone antagonist use in controlled ovarian stimulation and intrauterine insemination cycles in women with polycystic ovary syndrome. Fertil Steril. 2010; 93 (4): 1179-84.

15. Lee TH, Lin YH, Seow KM, Hwang JL, Tzeng CR, Yang YS. Effectiveness of cetrorelix for the prevention of premature luteinizing hormone surge during controlled ovarian stimulation using letrozole and gonadotropins: a randomized trial. Fertil Steril. 2008; 90 (1): 113-20.

16. Mitwally MF, Abdel-Razeq S, Casper RF. Human chorionic gonadotropin administration is associated with high pregnancy rates during ovarian stimulation and timed intercourse or intrauterine insemination. Reprod Biol Endocrinol. 2004; 2: 55-59.

17. Dickey RP, Taylor SN, Lu PY, Sartor BM, Rye PH, Pyrzak R. Risk factors for high-order multiple pregnancy and multiple birth after controlled ovarian hyperstimulation: results of 4,062 intrauterine insemination cycles. Fertil Steril. 2005; 3: 671-83.

18. Kaplan PF, Patel M, Austin DJ, Freund R. Assessing the risk of multiple gestation in gonadotropin intrauterine insemination cycles. Am J Obstet Gynecol. 2002; 6: 1244-7.

19. Martinez F, Barri PN, Coroleu B, et al. Women with poor response to IVF have lowered circulating gonadotrophin surge-attenuating factor (GnSAF) bioactivity during spontaneous and stimulated cycles. Hum Reprod. 2002; 3: $634-40$.

\section{Allahbadia Gautam}

Medical Director, Rotunda-The Center For Human Reproduction, Bandra, Mumbai For all correspondence: gautam@rotundaivf.com 